(2)

\section{OPEN ACCESS}

${ }^{1}$ St Helens and Knowsley Teaching Hospitals NHS Trust, Prescot, UK

${ }^{2}$ Stroke, St Helens and Knowsley Teaching Hospitals NHS Trust, Prescot, UK

Correspondence to Dr Fathalla Elnagi;

Fathalla.Elnagi@sthk.nhs.uk

Accepted 25 November 2021

Check for updates

(c) BMJ Publishing Group Limited 2021. Re-use permitted under CC BY-NC. No commercial re-use. See rights and permissions. Published by BMJ.

To cite: Davis E, Elnagi $F$ Smith T. BMJ Case Rep

2021:14:e245723.

doi:10.1136/bcr-2021-

245723

\title{
CT perfusion: stroke, seizure or both?
}

\author{
Elizabeth Davis, ${ }^{1}$ Fathalla Elnagi, ${ }^{2}$ Thomas Smith ${ }^{1}$
}

\section{SUMMARY}

An 88-year-old male with a history of hypertension, ischaemic heart disease and Bell's palsy presented with symptoms and signs of an acute ischaemic stroke. National Institutes of Health Stroke Scale (NIHSS) was 19 at presentation, indicative of potential large vessel occlusion. The initial CT scan revealed evidence of small vessel disease and arterial calcification. As there were no contraindications, he received thrombolytic treatment. CT angiography and CT perfusion imaging were performed in preparation for possible thrombectomy. There was no evidence of a large vessel thrombus, and changes on CT perfusion were suggestive of seizure activity, with relative hyperperfusion on the cerebral hemisphere of interest. Post thrombolysis, his NIHSS was 5. An MR scan revealed evidence of bilateral thalamic infarcts. After a period of rehabilitation, he was discharged home and independently mobile but with cognitive impairment. Acute stroke care increasingly uses multimodal imaging to confirm the clinical diagnosis and help optimise initial emergency management. Such imaging is useful in determining whether the presentation is a vascular event or stroke mimic. Moreover, seizures complicate and mimic acute strokes, which can lead to therapeutic uncertainty. This case highlights the increasingly sophisticated investigation of patients presenting with suspected acute stroke, with the attendant need for accurate interpretation by experienced clinicians.

\section{BACKGROUND}

Bilateral thalamic infarction is a rare phenomenon thought to account for around $0.6 \%$ of ischaemic strokes. ${ }^{1}$ The thalamus is a diencephalic structure with unique physiology in which neuronal firing patterns (tonic vs bursting) alter with physiological state and activity. It has a central role in the flow of information between the body and the brain, as well as the integration of information, and pathology affecting the thalamus can affect visual function, auditory pathways, somatosensory transmission, motor pathways, consciousness, and arousal and sleep. Depending on the vascular territory affected within the thalamus, the clinical syndromes differ and have been well described by Schmahmann. ${ }^{2}$

The thalamus is supplied by four major vessels: tuberothalamic, inferolateral, paramedian and posterior choroidal arteries. The most common aetiology of bilateral thalamic infarcts is cardioembolic, either via a tip of the basilar phenomenon (when other structures are usually involved) or in a patient with the artery of Percheron anatomical variant, described by the French neurologist, Percheron, in $1973 .^{3}$ In this variant, a single P1 segment of the posterior cerebral artery gives rise to blood vessels supplying both thalami. The variant is thought to be present in between $7 \%$ and $11 \%$ of people based on cadaveric studies, ${ }^{45}$ and thromboembolism of this vessel is the cause for ischaemic stroke in 4\%-18\% of all thalamic strokes. ${ }^{6}$

CT perfusion imaging of the brain is increasingly used in the context of acute ischaemic stroke although it is not available in all centres. While not required for decision-making in patients presenting with ischaemic stroke with a clear symptom onset time and within well-accepted time windows, it has a role in extending the therapeutic window for certain patients, improving diagnostic accuracy of stroke mimics, enhancing and expediting occlusion localisation in patients with more distal occlusions, and providing early prognostic information that can improve communication with patients and their relatives. ${ }^{7}$ The case we present had CT perfusion imaging inconsistent with the clinical picture, being suggestive of seizure activity, which is an uncommon but reported presentation of bilateral thalamic infarction, and as shown in the case we present, the CT perfusion imaging need not delay the initiation of thrombolysis where conventional indications remain. It can add extra information for the decision-making process as discussed below.

\section{CASE PRESENTATION}

A usually independent and active octogenarian with no pre-existing cognitive impairment and a preadmission Barthel Index of 20 presented to the emergency department of our institution. His medical history included angina, hypertension and a previous Bell's palsy affecting the left side of his face. Medication prior to admission included aspirin $75 \mathrm{mg}$ once a day, isosorbide mononitrate $40 \mathrm{mg}$ once a day, lisinopril $5 \mathrm{mg}$ once a day, artificial tears as required, omeprazole $20 \mathrm{mg}$ once a day, atenolol $25 \mathrm{mg}$ once a day and atorvastatin $20 \mathrm{mg}$ once a day.

He was last seen well at 10:00 but was found at 12:40 'confused', with speech disturbance associated with a right-sided facial droop and right-sided weakness. He arrived at 13:40 and the stroke team were contacted at 13:50, at which point his National Institutes of Health Stroke Scale (NIHSS) was 19: although he was conscious, he had severe aphasia (and therefore was unable to answer questions about the month and his age), he struggled to obey two commands, had visual inattention, significantly reduced power in both the right arm and leg, near unintelligible speech and severe hemi-inattention.

Intravenous access was obtained and baseline blood tests sent, all of which were unremarkable. Plain CT of the head revealed changes consistent with small vessel disease and arterial calcification particularly in the middle cerebral arteries. In view of the NIHSS score and symptoms and signs 
consistent with a left total anterior circulation ischaemic infarct, and in the absence of any contraindications, the patient was thrombolysed with intravenous alteplase 26 min from referral. As the thrombolytic therapy was being infused, we proceeded to CT angiography and CT perfusion, in preparation for possible thrombectomy of the suspected thrombus occluding the left middle cerebral artery.

Initially, the patient's blood pressure had been acceptable $(155 / 88 \mathrm{~mm} \mathrm{Hg})$, but this rose during thrombolysis and a bolus of intravenous labetalol was required to bring the blood pressure back within range. Thereafter, blood pressure control was adequate.

CT angiogram did not reveal any large vessel thrombus, and CT perfusion imaging using Brainomix software revealed changes compatible with seizure activity: relatively reduced time to peak (TTP) and time to maximum (Tmax) in the left cerebral hemisphere compared with the right, and increased relative cerebral blood volume (rBV) and relative cerebral blood flow $(\mathrm{rBF})$ on the left compared with the right. For readers unfamiliar with Brainomix software, it produces a number of useful outputs including visual colour maps of calculated perfusion parameters overlaid on the corresponding anatomical CT brain slice. It seeks to identify areas of perfusion mismatch and calculate relevant volumes of ischaemic core and penumbra, presenting those quantitatively and visually.

Forty-five minutes into thrombolysis, the patient deteriorated, becoming drowsy but rousable to voice and with difficulty clearing upper airway secretions. There were no convulsions and no eye deviations. Although a bleeding complication would be unlikely at this stage, a repeat plain CT of the head was performed, which confirmed no evidence of bleeding. The patient was transferred to our hyperacute stroke unit where he remained for 5 days before transfer to the stroke unit.

Four hours after thrombolysis, the patient was more alert: his NIHSS score had reduced to 5 (for mild-moderate aphasia, near unintelligible speech and the inability to answer the month or his age).

Initial speech and language assessments documented delayed processing, jargon and difficulty with expression. He had no insight into his deficits. A Montreal Cognitive Assessment at 48 hours had a score of $11 / 30$ with deficits in visuospatial abilities, attention, language, abstraction, delayed recall and orientation. Further therapy input commented on a gradual recovery of speech over the subsequent week, with dysphonia, perseveration, difficulty with recall of recent events, and some confusion and memory deficits.

The MRI demonstrated bilateral paramedian thalamic stroke, and the final diagnosis was bilateral paramedian thalamic stroke presenting as acute symptomatic seizure.

The patient was discharged on day 8 from the stroke unit with a return of physical function but cognitive deficits leaving him with a Barthel Index of 5 on discharge.

\section{INVESTIGATIONS}

Routine blood tests including full blood count, urea and electrolytes, coagulation screen, venous blood gas with lactate, liver function, cholesterol and HbA1c were performed in the emergency department at presentation and were unremarkable. An ECG demonstrated normal sinus rhythm at a rate of 97 beats per minute, with a single ventricular ectopic, partial right bundle branch block, normal axis and no overt ischaemia.

The plain CT of the head demonstrated moderate periventricular chronic ischaemia, with further ischaemic change in
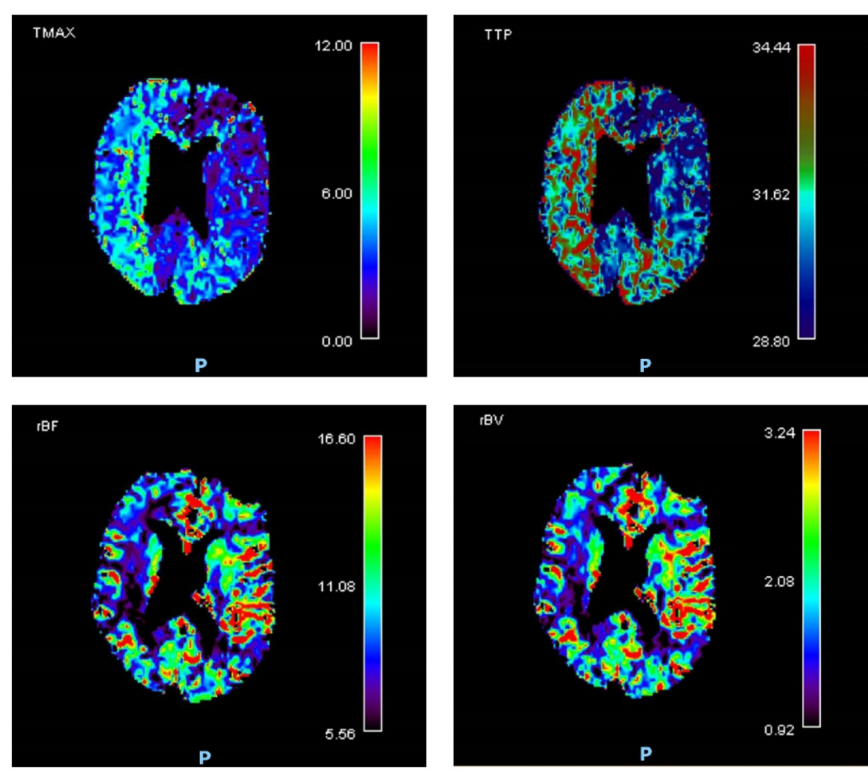

Figure 1 CT perfusion images within 30 min of presentation demonstrating reduced time to maximum (Tmax) and time to peak (TTP) in the left cerebral hemisphere compared with the right, and increased relative cerebral blood volume (rBV) and relative cerebral blood flow (rBF) on the left compared with the right.

both insular regions. No acute infarct was identified, but both internal carotid arteries were noted to be markedly calcified, with the calcification extending into the middle cerebral arteries. There was no evidence of intracranial haemorrhage or other abnormality.

CT angiography revealed no significant stenosis, occlusion or dissection in the carotid arteries. A dominant vertebral artery was noted, and the attenuated right anterior cerebral artery was supplied by the anterior communicating artery. The circle of Willis was otherwise felt to be normal.

CT perfusion showed a relatively reduced TTP and Tmax in the left cerebral hemisphere compared with the right, and increased $\mathrm{rBV}$ and $\mathrm{rBF}$ on the left compared with the right. All these parameters are in keeping with seizure activity affecting the left cerebral hemisphere (figure 1).

MRI of the head without contrast demonstrates hyperintensity of both thalami on axial diffusion-weighted imaging (DWI) with restricted diffusion on the apparent diffusion coefficient map. There is increased signal corresponding with the abnormalities on the DWI seen on the fluid-attenuated inversion recovery

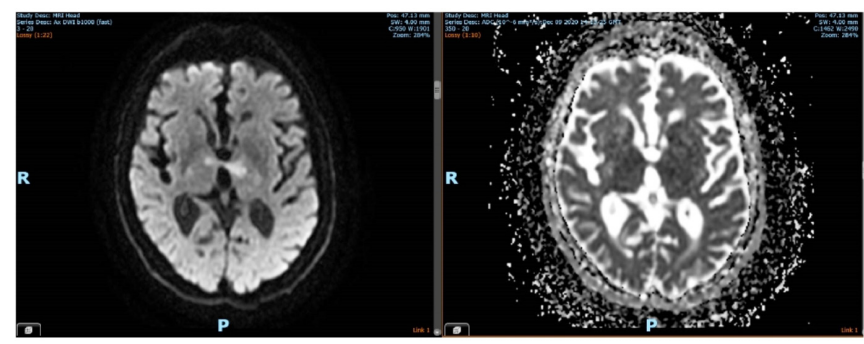

Figure 2 Uncontrasted MRI of the head demonstrating hyperintensity of both thalami on axial diffusion-weighted imaging (left-hand image) with restricted diffusion on the apparent diffusion coefficient map (right-hand image). 
images. This is in keeping with acute bilateral thalamic infarction (figure 2).

\section{DIFFERENTIAL DIAGNOSIS}

The initial presentation of our patient appeared consistent with a left total anterior circulation infarct. His dense hemiparesis, facial weakness, visual field deficit, hemi-inattention, dysarthria and dysphasia all pointed to an acute vascular event. Seizure secondary to an underlying intracranial abnormality was considered less likely in view of the persistent neurological findings with preserved alertness, and there was no history of epilepsy or witnessed epileptiform activity. As with all abrupt onset neurological conditions, hypoglycaemia was excluded by the ambulance crew pre-hospital and normoglycaemia confirmed in the emergency department. The abrupt symptom onset and absence of fever or prodrome made encephalitis and meningitis unlikely, as did the blood diagnostics which failed to demonstrate abnormal white blood cell count or significantly raised $\mathrm{C}$ reactive protein. Plain CT excluded any haemorrhage in the relevant vascular territory as well as any significant tumour.

There were no contraindications to thrombolysis, and on this basis we proceeded with the treatment.

Once the thrombolysis had started, the CT perfusion scan was completed and caused some doubt to be cast on the initial diagnosis. The left-sided hyperperfusion made the right cerebral hemisphere look relatively hypoperfused. The CT perfusion scan was more in keeping with a seizure, but in view of the persistent widespread neurological symptoms with no seizure activity witnessed and multiple vascular risk factors (age, sex, hypertension, coronary artery disease), the risk-benefit balance was favoured to continue to treat as ischaemic stroke, given that stroke can, in up to $4 \%$ of cases, present as seizures.

The final diagnosis was arrived at using a combination of imaging modalities-both CT perfusion and non-contrast MRI.

\section{TREATMENT}

The patient was initially thrombolysed with intravenous alteplase and went on to receive aspirin $300 \mathrm{mg}$ once a day for 14 days and then was switched to clopidogrel $75 \mathrm{mg}$ once a day thereafter in accordance with nationally agreed guidance on secondary prevention after stroke in the absence of atrial fibrillation. ${ }^{8}$ Levetiracetam was initially started for the treatment of acute symptomatic seizure.

\section{OUTCOME AND FOLLOW-UP}

The patient made a full motor recovery, but his cognition continued to be an ongoing concern. Following some intensive inpatient therapies, he was discharged to his own home to continue rehabilitation on day 8 after admission. Outpatient Holter monitoring and transthoracic echocardiography were performed in accordance with local guidelines looking for a cardioembolic source of stroke. A transthoracic echocardiogram revealed good left ventricular systolic function with an ejection fraction of more than $50 \%$, a normal right ventricle and mild mitral regurgitation with no evidence of intracardiac thrombus. Seven-day Holter monitoring showed normal sinus rhythm throughout with no significant arrhythmia or pauses. The patient will be seen in our stroke follow-up clinic in due course.

\section{DISCUSSION}

CT perfusion is increasingly used in the assessment of patients presenting with suspected acute stroke and can be beneficial in excluding conditions that mimic stroke such as seizures and posterior reversible encephalopathy syndrome, which otherwise might be treated with thrombolysis. Reported changes seen on CT perfusion with various stroke mimics can at times appear odd. ${ }^{9}$ Typical changes of seizures on CT perfusion scan include ipsilateral increased perfusion, or contralateral increased TTP, or unilateral increased cerebral blood flow and decreased TTP. In one review, 91\% of patients presenting with seizure to an emergency department over a 4-year period had an abnormal CT perfusion scan, with $66 \%$ having the typical changes described above. ${ }^{10}$ Seizures typically produce perfusion abnormalities that do not respect vascular territories, and often there is increased cerebral blood flow ictally, decreased flow post-ictally and then a normalisation.

Our patient's scan showed hyperperfusion of the left cerebral hemisphere. Hyperperfusion is seen more commonly when perfusion scanning is performed within the first 3 hours of the event, ${ }^{11}$ and post-ictal changes can be unihemispheric and typically contralateral to the clinical deficits ${ }^{10}$ as in our case. Although in this case the thrombolysis bolus dose was given prior to CT angiography and CT perfusion scanning, it is extremely unlikely to be the cause of the abnormalities demonstrated on our patient's perfusion map: opening a large artery should not cause hyperperfusion, only normal perfusion. This supposition is borne out with the final clinical and radiological findings that confirm the diagnosis of bilateral thalamic stroke.

The European Stroke Organisation (ESO) 2021 guidance on intravenous thrombolysis ${ }^{12}$ specifically considers the question of thrombolysis for patients presenting with seizure at stroke onset within 4.5 hours of onset of ischaemic stroke symptoms. Although seizure can be the presenting feature of stroke, this subgroup of patients have traditionally been excluded from randomised controlled trials for thrombolysis for a variety of reasons, including concerns around thrombolysing a stroke mimic or about occult head injury acquired during a seizure-and therefore attendant increased risk of intracranial haemorrhage. The ESO guidance makes the following weak recommendation: 'for patients with acute ischaemic stroke of $<4.5$ hours' duration who have seizures at time of stroke onset, and for whom there is no suspicion of a stroke mimic or significant head trauma, we suggest intravenous thrombolysis with alteplase'. ${ }^{12}$ This recommendation is based on observational studies suggesting that the risk of symptomatic intracranial haemorrhage following thrombolysis of a stroke mimic is low, ${ }^{13} 14$ at around 1\%, compared with $7.9 \%$ in acute ischaemic stroke. ${ }^{14}$ In addition, the number needed to treat for thrombolysis is 10 if administered within 3 hours/with a favourable CT/CT perfusion imaging, and seizure at onset of acute ischaemic stroke is not an independent predictor of poor prognosis. ${ }^{15}$

Advanced imaging methods such as CT perfusion or MRI with DWI and apparent diffusion coefficients have not been used in the context of seizures and suspected acute stroke in randomised controlled trials, and their use has the potential to complicate decision-making as our case demonstrates. The major point for discussion in the management of our patient revolves around whether the CT perfusion should have prevented us from thrombolysing the patient, who had in fact suffered bilateral ischaemic thalamic infarcts. The evidence is weak, but the risk of harm was perceived to be low, and the current guidance would favour thrombolysis in the absence of underlying structural brain abnormality and metabolic or infectious stroke mimic. Because advanced imaging techniques have a role in recognising stroke mimics, ${ }^{9}$ many stroke centres have incorporated them into their standard assessment protocols. This case demonstrates the importance of clinical assessment alongside 
the use of CT perfusion imaging: if the CT perfusion alone had been considered, this male would not have received intravenous thrombolysis and would have probably been left significantly more disabled following his bilateral thalamic infarcts. Given the current evidence, withholding intravenous thrombolysis from a patient with presumed ischaemic stroke but seizure at onset seems difficult to justify. Acute MRI with DWI could have saved this particular diagnostic conundrum but is not feasible in many centres nationally.

As with so many technologies in medicine, CT perfusion has the potential to bring great benefit, but only when used by experienced clinicians with an understanding of its limitations.

\section{Learning points}

- Thalamic infarcts can present as seizures

- CT perfusion has a role in helping define aetiology, but clinical acumen is vital for its interpretation and application in clinical practice.

- MRI is a helpful tool in establishing the diagnosis of acutel subacute stroke, especially where there is clinical uncertainty.

Correction notice This article has been corrected since published online. The spelling of author's name has been corrected from "Elizebath Davies" to "Elizabeth Davis".

Contributors ED was the registrar on-call when the patient arrived. When the diagnosis became clear after performing MRI brain, we had a lengthy discussion regarding the imaging interpretation and clinical presentation of the patient, and she then showed her interest in writing the case. She wrote the case under FE's guidance and updated the case report with his advice to meet the BMJ case report. All authors discussed the results and contributed to the final manuscript. FE was the stroke physician who looked after the patient, confirmed the final diagnosis, reviewed the case report and guided ED during the writing. He spoke to the patient and obtained written consent. He provided critical feedback and helped shape the case report, analysis and manuscript. TS was the physician on-call on the patient's arrival. He was involved in reviewing the case report and provided critical feedback.

Funding This study was funded by St Helens and Knowsley Teaching Hospital NHS Trust (839267).

Competing interests None declared.

Patient consent for publication Consent obtained directly from patient(s)
Provenance and peer review Not commissioned; externally peer reviewed.

Open access This is an open access article distributed in accordance with the Creative Commons Attribution Non Commercial (CC BY-NC 4.0) license, which permits others to distribute, remix, adapt, build upon this work non-commercially, and license their derivative works on different terms, provided the original work is properly cited and the use is non-commercial. See: http://creativecommons.org/ licenses/by-nc/4.0/.

Case reports provide a valuable learning resource for the scientific community and can indicate areas of interest for future research. They should not be used in isolation to guide treatment choices or public health policy.

\section{REFERENCES}

1 Kumral E, Evyapan D, Balkir K, et al. Bilateral thalamic infarction. Clinical, etiological and MRI correlates. Acta Neurol Scand 2001;103:35-42.

2 Schmahmann JD. Vascular syndromes of the thalamus. Stroke 2003:34:2264-78.

3 Percheron $\mathrm{G}$. The anatomy of the arterial supply of the human thalamus and its use for the interpretation of the thalamic vascular pathology. Z Neurol 1973;205:1-13.

$4 \mathrm{Uz}$ A. Variations in the origin of the thalamoperforating arteries. J Clin Neurosci 2007:14:134-7.

5 Kocaeli H, Yilmazlar S, Kuytu T, et al. The artery of Percheron revisited: a cadaveric anatomical study. Acta Neurochir 2013;155:533-9.

6 Lazzaro NA, Wright B, Castillo M, et al. Artery of percheron infarction: imaging patterns and clinical spectrum. AJNR Am J Neuroradiol 2010;31:1283-9.

7 Demeestere J, Wouters A, Christensen $\mathrm{S}$, et al. Review of perfusion imaging in acute ischemic stroke: from time to tissue. Stroke 2020;51:1017-24.

8 Rudd AG, Bowen A, Young G, et al. National clinical guideline for stroke: 5 th edition 2016. clinical medicine 2017

9 Gonzalez-Martinez A, Trillo S, Benavides-Bernaldo de Quirós C, et al. Predictors of perfusion computed tomography alterations in stroke mimics attended as stroke code. Eur J Neurol 2021;28:1939-48.

10 Khaku A, Hedna V, Waters M. Utility of CT perfusion (CTP) imaging in seizures: a retrospective analysis and literature review. Neurology 2014;82.

11 Payabvash S, Oswood MC, Truwit CL, et al. Acute CT perfusion changes in seizure patients presenting to the emergency department with stroke-like symptoms: correlation with clinical and electroencephalography findings. Clin Radiol 2015;70:1136-43.

12 Berge E, Whiteley W, Audebert $\mathrm{H}$, et al. European stroke organisation (ESO) guidelines on intravenous thrombolysis for acute ischaemic stroke. Eur Stroke J 2021;6:I-LXII.

13 Tsivgoulis G, Zand R, Katsanos AH, et al. Safety of intravenous thrombolysis in stroke mimics: prospective 5-year study and comprehensive meta-analysis. Stroke 2015;46:1281-7.

14 Zinkstok SM, Engelter ST, Gensicke H, et al. Safety of thrombolysis in stroke mimics. Stroke 2013;44:1080-4.

15 Polymeris AA, Curtze $\mathrm{S}$, Erdur $\mathrm{H}$, et al. Intravenous thrombolysis for suspected ischemic stroke with seizure at onset. Ann Neurol 2019;86:770-9.

Copyright 2022 BMJ Publishing Group. All rights reserved. For permission to reuse any of this content visit

https://www.bmj.com/company/products-services/rights-and-licensing/permissions/

BMJ Case Report Fellows may re-use this article for personal use and teaching without any further permission.

Become a Fellow of BMJ Case Reports today and you can:

- Submit as many cases as you like

- Enjoy fast sympathetic peer review and rapid publication of accepted articles

- Access all the published articles

- Re-use any of the published material for personal use and teaching without further permission

Customer Service

If you have any further queries about your subscription, please contact our customer services team on +44 (0) 2071111105 or via email at support@bmj.com.

Visit casereports.bmj.com for more articles like this and to become a Fellow 